\title{
Caractéristiques cliniques des cas pédiatriques de coqueluche au Québec, 2015 à 2017
}

\author{
M Desjardins 1,4 , D lachimov², S Mousseau ${ }^{3}$, P Doyon-Plourde ${ }^{1,4}$, N Brousseau ${ }^{5}$, F Rallu 6 , \\ C Quach ${ }^{1,4,6,7,8 *}$
}

\section{Résumé}

Contexte : L'introduction du vaccin anticoquelucheux acellulaire pourrait avoir modifié les caractéristiques épidémiologiques et cliniques de la coqueluche chez les enfants canadiens.

Objectif : Décrire les données démographiques, les manifestations clinique et les issues cliniques chez les enfants et les adolescents atteints de coqueluche s'étant présentés dans un hôpital de soins tertiaires.

Méthodologie : Étude de cohorte rétrospective menée chez des patients consécutifs examinés au Centre hospitalier universitaire Sainte-Justine (CHUSJ) et soumis à une recherche par amplification en chaîne par polymérase (polymerase chain reaction [PCR]) multiplex en temps réel pour les bactéries Bordetella pertussis ou B. parapertussis entre juin 2015 et mars 2017. Les données démographiques, les manifestations cliniques et les issues ont été décrites pour les résultats positifs. L'échelle modifiée de Preziosi a servi à évaluer la gravité de la maladie; une forme grave de la maladie correspond à un score de sept et plus.

Résultats : La distribution par âge des 144 patients dont les résultats de PCR étaient positifs et qui ont été reçus en consultation au CHUSJ s'établissait comme suit : moins de trois mois ( $n=25 / 144,17,4 \%)$, de quatre mois à neuf ans $(n=63 / 144,43,8 \%)$ et de dix à 18 ans ( $n=56 / 144,38,9 \%$ ). Les symptômes les plus courants comprenaient la toux paroxystique $(70,1 \%)$, la toux émétisante $(47,2 \%)$ et la rhinorrhée $(33,3 \%)$. Chez les nourrissons de moins de trois mois, plus de $84,0 \%$ des cas consistaient en une coqueluche grave $(92,0 \%$ ont nécessité une hospitalisation et $28,0 \%$ ont été admis aux soins intensifs). Chez les enfants de quatre mois à neuf ans, 22,2\% étaient atteints d'une coqueluche grave et 11,1\% ont été hospitalisés. Seulement deux $(3,6 \%)$ enfants de plus de dix ans présentaient une forme grave de la maladie.

Conclusion : La coqueluche touche encore des enfants de tous âges au Québec. Chez les enfants plus âgés, la maladie est plus souvent bénigne. Chez les nourrissons qui ne bénéficient pas encore de la protection complète offerte par la vaccination, la maladie est souvent grave, en particulier chez ceux de moins de trois mois. Ces données probantes viennent appuyer la mise en œuvre d'un programme de vaccination contre la coqueluche chez les femmes enceintes.

\author{
Affiliations \\ ${ }^{1}$ Microbiologie médicale et \\ infectiologie, Centre Hospitalier \\ de l'Université de Montréal, \\ Montréal (Québec) \\ ${ }^{2}$ Centre de recherche, Centre \\ Hospitalier Universitaire \\ Sainte-Justine, Montréal (Québec) \\ ${ }^{3}$ Service d'urgence pédiatrique, \\ Département de pédiatrie, \\ Centre Hospitalier Universitaire \\ Sainte-Justine, Montréal (Québec) \\ ${ }^{4}$ Département de microbiologie, \\ infectiologie et immunologie, \\ Faculté de médecine, Université \\ de Montréal, Montréal (Québec) \\ ${ }^{5}$ Direction des risques \\ biologiques et de la santé au \\ travail, Institut national de santé \\ publique du Québec, Québec \\ (Québec) \\ ${ }^{6}$ Département de microbiologie \\ médicale, Centre Hospitalier \\ Universitaire Sainte-Justine, \\ Montréal (Québec) \\ ${ }^{7}$ Prévention et contrôle des \\ infections, Centre Hospitalier \\ Universitaire Sainte-Justine, \\ Montréal (Québec) \\ ${ }^{8}$ Présidente, Comité consultatif \\ national de l'immunisation
}

*Correspondance : c.quach@ umontreal.ca

\begin{abstract}
Citation proposée : Desjardins M, lachimov D, Mousseau S, Doyon-Plourde P, Brousseau N, Rallu F, Quach C. Caractéristiques cliniques des cas pédiatriques de coqueluche au Québec, 2015 à 2017. Relevé des maladies transmissibles au Canada 2018;44(9):214-20. https://doi.org/10.14745/ccdr.v44i09a01f
\end{abstract}

Mots-clés : coqueluche, pédiatrie, symptômes, issues, Québec

\section{Introduction}

La coqueluche est une infection des voies respiratoires causée par les bactéries Bordetella pertussis et B. parapertussis. Les patients qui en sont atteints peuvent manifester un large éventail de symptômes en fonction de l'âge, de l'état vaccinal et des co-infections, ce qui complique souvent le diagnostic $(1,2)$.

La coqueluche est une maladie évitable par la vaccination. La vaccination contre la coqueluche avec vaccin entier, dont I'introduction au Canada remonte à 1943, a entraîné une baisse significative de l'incidence de la maladie (3). Le vaccin entier a été remplacé par un vaccin acellulaire à la fin des années 1990 afin de réduire l'incidence des événements indésirables suivant l'immunisation.

De nos jours, le vaccin contenant la valence coquelucheuse est administré à l'âge de deux, quatre, six et dix-huit mois, suivi d'un rappel entre quatre et six ans. Au Québec, le programme de vaccination acellulaire universel a été lancé en 1998, entraînant 
un effet notable sur l'incidence de la coqueluche (4-6). En 2016, malgré une couverture vaccinale de l'ordre de $97,3 \%$ des enfants à un an (7), l'incidence de la coqueluche chez les moins de 18 ans s'élevait encore à 60 cas par 100000 enfants (8).

Il a été démontré que l'immunité et la protection que confère le vaccin acellulaire diminuent rapidement (9), ce qui pourrait avoir modifié les manifestations cliniques de la coqueluche chez les enfants. Les jeunes bébés sont particulièrement vulnérables à la coqueluche, car les nourrissons de moins de trois mois n'ont reçu qu'une seule dose du vaccin anticoquelucheux, ce qui ne procure qu'une protection partielle $(6,10,11)$. Pour remédier à cette situation, le Comité consultatif national de l'immunisation $(\mathrm{CCNI})$ a évalué les données probantes relatives à la vaccination des femmes enceintes et a déterminé qu'il s'agissait d'un moyen de prévention hautement efficace contre la coqueluche chez les nourrissons (12-14). En 2018, le CCNI a recommandé I'immunisation des femmes enceintes contre la coqueluche (15), soulignant qu'une telle mesure pourrait mener à une réduction de $90 \%$ des cas de coqueluche chez les nourrissons nés de mères vaccinées (16).

Les dernières études en milieu hospitalier décrivant les caractéristiques épidémiologiques et cliniques de la coqueluche chez les enfants canadiens ont été menées entre 1991 et 2004

$(17,18)$; par conséquent, le fardeau actuel de la maladie chez les enfants au Canada n'est pas connu.

L'objectif de la présente étude consistait à décrire les manifestations cliniques et les issues chez les enfants ayant contracté la coqueluche et qui ont été évalués entre juin 2015 et mars 2017 au Centre Hospitalier Universitaire Sainte-Justine (CHUSJ). Cet hôpital est situé à Montréal (Québec) et il s'agit du seul hôpital autonome pour enfants de la province du Québec, avec près de 80000 consultations au service d'urgence par année (19).

\section{Méthodologie}

\section{Méthodologie de l'étude}

Il s'agissait d'une étude d'observation rétrospective de cohorte menée chez des patients consécutifs évalués au CHUSJ pour un cas soupçonné de coqueluche. Les enfants présentant un cas soupçonné de coqueluche provenaient de la circonscription hospitalière et ont été évalués pour la plupart par le service d'urgence. Certains enfants ont été évalués pour la coqueluche durant leur hospitalisation. Tous les enfants ont été soumis à un test d'amplification en chaîne par polymérase (polymerase chain reaction [PCR]) multiplex de bactéries (B. pertussis, B. parapertussis, B. holmesii, Mycoplasma pneumoniae et Chlamydophila pneumoniae). Depuis 2015, tous les cas soupçonnés de coqueluche observés au CHUSJ sont testés à l'aide du PCR multiplex.

Les cas ayant obtenu un résultat positif pour $B$. pertussis ou B. parapertussis entre juin 2015 et mars 2017 ont été identifiés dans le système d'information du laboratoire et les données cliniques ont été extraites au moyen d'un examen manuel des dossiers. Le protocole de l'étude a été approuvé par le comité d'éthique du CHUSJ.

\section{Population de l'étude}

L'étude a inclus des patients consécutifs âgés de zéro à 17 ans dont le test de PCR multiplex s'est révélé positif (valeur du cycle seuil, ou cycle threshold [Ct], inférieure à 36) pour B. pertussis ou $B$. parapertussis, et dont les données cliniques et de laboratoire étaient accessibles dans le système d'information du laboratoire de microbiologie du CHUSJ entre juin 2015 et mars 2017. Comme la bactérie $B$. parapertussis peut causer une maladie semblable à la coqueluche et que le vaccin actuel contre $B$. pertussis pourrait offrir une protection croisée contre $B$. parapertussis (20), les patients dont les PCR ont révélé la présence de $B$. parapertussis ont été inclus dans l'étude. Les patients affichant des résultats de PCR équivoques ( $\mathrm{Ct}$ de 36 à 39,9 ) ont également été retenus pour l'étude, puisqu'ils sont actuellement considérés comme des cas de coqueluche par les autorités de santé publique du Québec lorsqu'ils présentent des symptômes compatibles à cette infection (6). Les patients ayant obtenu un résultat positif pour $B$. holmesii n'ont pas été retenus, car l'espèce Bordetella peut entraîner une maladie significativement différente (1). Les patients âgés d'au moins 18 ans ainsi que les patients qui n'ont pas été reçus en consultation clinique au CHUSJ (p. ex. des échantillons reçus d'autres hôpitaux) ont été exclus.

\section{Collecte et analyse des données}

En plus de l'analyse des données de laboratoire, un examen manuel des dossiers médicaux électroniques a été effectué (à I'aide de Chartmaxx; Quest Diagnostics, Secaucus, État du New Jersey, États-Unis [É-U]), au moyen des fiches d'observation normalisées pour recueillir de l'information sur 1) les manifestations cliniques (à l'aide de l'évaluation des infirmières de triage et des notes cliniques des médecins le jour où la PCR a été demandée), 2) les résultats de l'investigation et 3 ) les issues cliniques (hospitalisation, durée du séjour, traitement aux macrolides, admission aux soins intensifs ou décès). La collecte de données a été effectuée par deux membres de l'équipe de recherche $(\mathrm{MD}, \mathrm{DI})$ et $10 \%$ des dossiers ont été analysés par les deux chercheurs afin d'évaluer la concordance inter-évaluateurs (établie à l'aide d'un test statistique kappa). Les patients ont été divisés en trois groupes d'âge, comme il a été suggéré au cours de la table ronde Global Pertussis Initiative tenue en février 2011 (21) : trois mois ou moins; de quatre mois à neuf ans; et de dix à dix-huit ans. Les nombres absolus et les proportions ont été utilisés pour analyser les données démographiques, les manifestations cliniques et les issues cliniques. Un écart interquartile (EIQ) a servi à évaluer la dispersion statistique des variables continues. L'échelle modifiée de Preziosi (ÉMP) (22) a été utilisée pour déterminer la gravité de la maladie. Un score supérieur ou égal à sept sur l'ÉMP correspond à une forme grave de coqueluche. Le tableur Microsoft Excel 2016 (Redmond, État de Washington, É-U) a été utilisé pour calculer les proportions et I'EIQ. Les analyses statistiques étaient descriptives $(23,24)$.

\section{Résultats}

Parmi les 1526 tests de PCR multiplex effectués entre le 11 juin 2015 et le 31 mars 2017, 173 patients ont affiché un résultat positif ou équivoque pour $B$. pertussis ou $B$. parapertussis (taux de positivité de 11,3\%). Vingt-neuf patients 
ont été exclus : deux d'entre eux étaient âgés de plus de 18 ans et 27 n'ont pas été reçus en consultation clinique au CHUSJ.

\section{Données démographiques}

Un total de 144 patients a été soumis à une analyse : 133 cas de $B$. pertussis (109 résultats positifs, 24 résultats équivoques); et 11 cas de $B$. parapertussis (sept résultats positifs, quatre résultats équivoques) (tableau 1). Les patients ont été regroupés pour I'analyse, en raison du petit nombre de patients ayant obtenu un résultat positif pour $B$. parapertussis et parce que ces deux bactéries provoquent des syndromes respiratoires semblables.

Tableau 1 : Caractéristiques des enfants atteints d'une coqueluche confirmée par PCR

\begin{tabular}{|l|r|r|r|r|}
\hline \multirow{2}{*}{ Caractéristiques } & \multicolumn{4}{|c|}{ Groupes d'âge, n (\%) } \\
\cline { 2 - 5 } & $\begin{array}{c}\text { 0 à 3 } \\
\text { mois }\end{array}$ & $\begin{array}{c}\text { 4 mois à } \\
\mathbf{9} \text { ans }\end{array}$ & $\begin{array}{c}10 \text { à } 18 \\
\text { ans }\end{array}$ & \multicolumn{1}{c|}{ Total } \\
\hline $\begin{array}{l}\text { Résultat de } \\
\text { laboratoire }\end{array}$ & $25(17,4)$ & $63(43,8)$ & $56(38,9)$ & $144(100,0)$ \\
\hline $\begin{array}{l}\text { Positif pour } B . \\
\text { pertussis }\end{array}$ & $19(76,0)$ & $45(71,4)$ & $45(80,4)$ & $109(75,7)$ \\
\hline $\begin{array}{l}\text { Positif pour B. } \\
\text { parapertussis }\end{array}$ & $1(4,0)$ & $5(7,9)$ & $1(1,8)$ & $7(4,9)$ \\
\hline $\begin{array}{l}\text { Équivoque pour B. } \\
\text { pertussis }\end{array}$ & $5(20,0)$ & $9(14,3)$ & $10(17,9)$ & $24(16,7)$ \\
\hline $\begin{array}{l}\text { Équivoque pour B. } \\
\text { parapertussis }\end{array}$ & $0(0,0)$ & $4(6,3)$ & $0(0,0)$ & $4(2,8)$ \\
\hline Sexe féminin & $14(56,0)$ & $33(52,4)$ & $35(62,5)$ & $82(56,9)$ \\
\hline Antécédents médicaux & \multicolumn{4}{|c|}{} \\
\hline Asthme & $0(0,0)$ & $11(17,5)$ & $11(19,6)$ & $22(15,3)$ \\
\hline Vaccination à jour & $22(88,0)$ & $42(66,7)$ & $48(85,7)$ & $112(77,8)$ \\
\hline Prématurité & $4(16,0)$ & $4(6,3)$ & $1(1,8)$ & $9(6,3)$ \\
\hline
\end{tabular}

Abréviations : B., Bordetella; $n$, nombre; PCR, amplification en chaîne par polymérase (polymerase chain reaction)

Parmi les 144 enfants, 25 (17,4 \%) avaient moins de trois mois, $63(43,8 \%)$ étaient âgés de quatre mois à neuf ans et $56(38,9 \%)$ avaient entre 10 et 18 ans. La proportion de tests positifs augmentait avec l'âge, pour atteindre un pic de 35 à $45 \%$ chez les adolescents de 10 à 15 ans (figure 1).

Figure 1 : Distribution par âge des patients ayant des résultats de $P C R$ positifs pour la coqueluche $(n=144)$

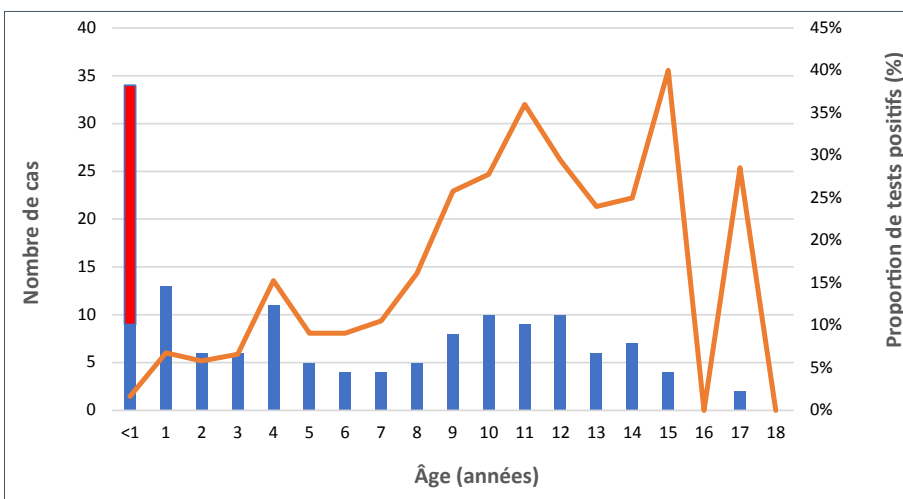

Nombre de cas Cas chez les nourrissons de moins de 3 mois _- Proportion de tests positifs Abréviation : PCR, amplification en chaîne par polymérase (polymerase chain reaction)
La coqueluche était déclarée tout au long de l'année (figure 2).

Figure 2 : Distribution des patients ayant des résultats de PCR positifs pour la coqueluche pendant la période de l'étude $(n=144)$

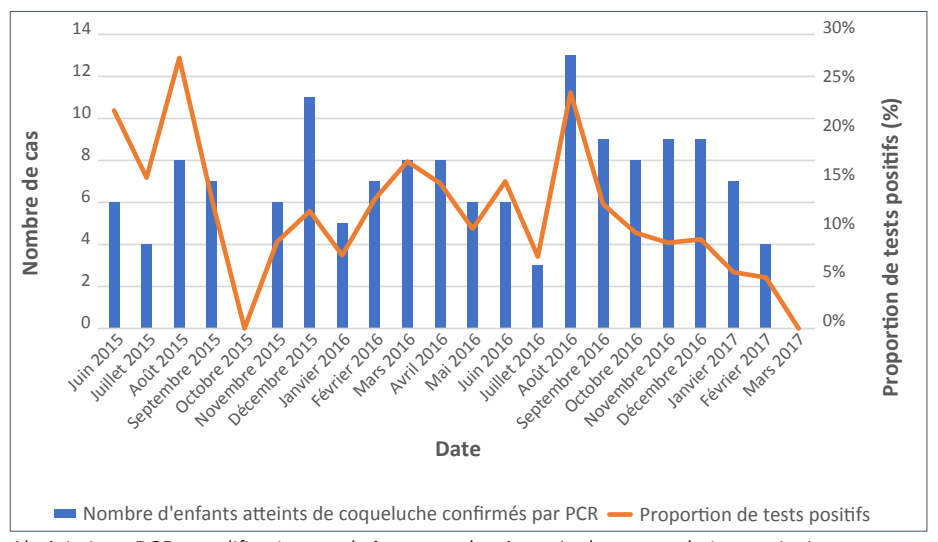

Abréviation : PCR, amplification en chaîne par polymérase (polymerase chain reaction)

\section{Manifestations cliniques}

Les symptômes les plus courants comprenaient la toux paroxystique $(70,1 \%)$, la toux émétisante $(47,2 \%)$ et la rhinorrhée (33,3\%) (tableau 2). Des 100 radiographies pulmonaires effectuées $(69,4 \%$ de tous les cas), seulement huit indiquaient la présence d'une pneumonie. Les 25 enfants de moins de trois mois étaient les plus gravement atteints par la coqueluche, avec un score médian de 12 à l'ÉMP (EIO : 9-15). En tout, $84,0 \%$ des enfants de moins de trois mois présentaient une forme grave de la maladie. Les 10 cas d'apnée, 75,0\% ( $n=15 / 20)$ des cas de cyanose, $76,9 \%(n=10 / 13)$ des cas de tirage et $45 \%(n=9 / 20)$ des cas de chant du coq appartenaient à ce groupe d'âge. Les enfants âgés de quatre mois à neuf ans étaient aussi nettement touchés : $22,2 \%$ présentaient une forme grave de la coqueluche. À titre comparatif, seulement deux $(3,6 \%)$ enfants de plus de 10 ans présentaient une coqueluche grave. La concordance inter-évaluateurs établie à l'aide d'une analyse statistique kappa s'élevait à 0,86 , ce qui démontre la bonne validité du processus de collecte de données.

Tableau 2 : Manifestations cliniques et examens paracliniques des enfants atteints d'une coqueluche confirmée par PCR

\begin{tabular}{|l|r|r|r|r|}
\hline \multirow{2}{*}{ Caractéristiques } & \multicolumn{5}{|c|}{ Groupes d'âge, n (\%) } \\
\cline { 2 - 5 } & $\begin{array}{r}\text { 0 à 3 } \\
\text { mois } \\
n=25\end{array}$ & $\begin{array}{c}\text { 4 mois à } \\
9 \text { ans } \\
n=63\end{array}$ & $\begin{array}{c}10 \text { à 18 } \\
\text { ans } \\
n=56\end{array}$ & Total \\
Manifestations cliniques \\
\hline Toux paroxystique & $20(80,0)$ & $47(74,6)$ & $34(60,7)$ & $101(70,1)$ \\
\hline Chant du coq & $9(36,0)$ & $7(11,1)$ & $4(7,1)$ & $20(13,9)$ \\
\hline Toux émétisante & $10(40,0)$ & $32(50,8)$ & $26(46,4)$ & $68(47,2)$ \\
\hline Cyanose & $15(60,0)$ & $4(6,3)$ & $1(1,8)$ & $20(13,9)$ \\
\hline Tirage & $10(40,0)$ & $3(4,8)$ & $0(0,0)$ & $13(9,0)$ \\
\hline Fièvre & $2(8,0)$ & $10(15,9)$ & $4(7,1)$ & $16(11,1)$ \\
\hline Rhinorrhée & $14(56,0)$ & $20(31,8)$ & $14(25,0)$ & $48(33,3)$ \\
\hline
\end{tabular}


Tableau 2 (suite) : Manifestations cliniques et examens paracliniques des enfants atteints d'une coqueluche confirmée par PCR

\begin{tabular}{|c|c|c|c|c|}
\hline \multirow{3}{*}{ Caractéristiques } & \multicolumn{4}{|c|}{ Groupes d'âge, n (\%) } \\
\hline & $\begin{array}{l}0 \text { à } 3 \\
\text { mois }\end{array}$ & $\begin{array}{l}4 \text { mois } \\
\text { à } 9 \text { ans }\end{array}$ & $\begin{array}{c}10 \text { à } 18 \\
\text { ans }\end{array}$ & Total \\
\hline & $n=25$ & $n=63$ & $n=56$ & $n=144$ \\
\hline \multicolumn{5}{|c|}{ Manifestations clinique (suite) } \\
\hline $\begin{array}{l}\text { Auscultation } \\
\text { pulmonaire } \\
\text { anormale }\end{array}$ & $8(32,0)$ & $8(12,7)$ & $2(3,6)$ & $18(12,5)$ \\
\hline Apnée & $10(40,0)$ & $0(0,0)$ & $0(0,0)$ & $10(6,9)$ \\
\hline Otite & $2(8,0)$ & $6(9,5)$ & $2(3,6)$ & $10(6,9)$ \\
\hline Pharyngite & $2(8,0)$ & $2(3,2)$ & $7(12,5)$ & $11(7,6)$ \\
\hline $\begin{array}{l}\text { Hémorragie sous- } \\
\text { conjonctivale }\end{array}$ & $0(0,0)$ & $1(1,6)$ & $0(0,0)$ & $1(0,7)$ \\
\hline Convulsions & $0(0,0)$ & $0(0,0)$ & $0(0,0)$ & $0(0,0)$ \\
\hline \multicolumn{5}{|l|}{ ÉMP } \\
\hline Moyenne & 11,8 & 4,7 & 3,3 & 5,4 \\
\hline Médiane & 12 & 5 & 3 & 5 \\
\hline - EIQ 25-75 & $9,0-15,0$ & $3,0-6,0$ & $2,0-5,0$ & $3,0-7,0$ \\
\hline $\begin{array}{l}\text { Cas graves } \\
\text { (ÉMP } \geq 7 \text { ) }\end{array}$ & $21(84,0)$ & $12(22,2)$ & $2(3,6)$ & $37(25,7)$ \\
\hline \multicolumn{5}{|l|}{ Examens paracliniques } \\
\hline $\begin{array}{l}\text { Détection d'agents } \\
\text { viraux par PCR } \\
\text { multiplex }\end{array}$ & $13(52,0)$ & $6(9,5)$ & $5(8,9)$ & $24(16,7)$ \\
\hline $\begin{array}{l}\text { - Virus respiratoire } \\
\text { détectéb }^{b}\end{array}$ & $5(38,5)$ & $2(33,3)$ & $1(20,0)$ & $8(33,3)$ \\
\hline $\begin{array}{l}\text { Formule sanguine } \\
\text { complète effectuée }\end{array}$ & $21(84,0)$ & $10(15,9)$ & $3(5,4)$ & $34(23,6)$ \\
\hline - Lymphocytose ${ }^{b}$ & $10(47,7)$ & $3(30,0)$ & $0(0,0)$ & $13(38,2)$ \\
\hline $\begin{array}{l}\text { Radiographie } \\
\text { pulmonaire } \\
\text { effectuée }\end{array}$ & $20(80,0)$ & $43(68,3)$ & $37(66,1)$ & $100(69,4)$ \\
\hline $\begin{array}{l}\text { - Pneumonie } \\
\text { décelée }^{b}\end{array}$ & $0(0,0)$ & $6(14,0)$ & $2(5,4)$ & $8(8,0)$ \\
\hline
\end{tabular}

Abréviations : ElQ, écart interquartile; ÉMP, échelle modifiée de Preziosi; n, nombre; $\geq$, supérieur ou égal à

Tous les résultats sont donnés en fonction de $\mathrm{n}(\%)$, exception faite de la moyenne ÉMP, de la médiane ÉMP (et EIQ)

${ }^{\text {b }}$ Ces pourcentages ont été calculés en fonction du nombre d'essais respectifs effectués

\section{Issues cliniques}

En tout, 20,8 \% des patients ont été hospitalisés (tableau 3). Les bébés de moins de trois mois présentaient le risque d'hospitalisation le plus élevé (92\%), en plus d'une proportion importante nécessitant une admission aux soins intensifs (28\%). À titre de comparaison, seulement $11 \%$ des enfants âgés de quatre mois à neuf ans ont été hospitalisés et aucun des enfants plus âgés. La majorité des patients $(75,2 \%)$ ont reçu un traitement aux macrolides. Aucun décès n'a été recensé.
Tableau 3 : Issues cliniques des enfants atteints d'une coqueluche confirmée par PCR

\begin{tabular}{|l|r|r|r|r|}
\hline \multirow{2}{*}{$\begin{array}{c}\text { Issues } \\
\text { cliniques }\end{array}$} & $\begin{array}{c}\text { 0 à 3 } \\
\text { mois } \\
\mathrm{n}=25\end{array}$ & $\begin{array}{c}\text { 4 mois à 9 } \\
\text { ans } \\
\mathrm{n}=63\end{array}$ & $\begin{array}{c}10 \text { à } 18 \\
\text { ans } \\
\mathrm{n}=56\end{array}$ & \multicolumn{1}{c|}{ Total } \\
\cline { 2 - 5 } & $23(92,0)$ & $7(11,1)$ & $0(0,0)$ & $30(20,8)$ \\
\hline Hospitalisation & 11,0 & 3,0 & 0,0 & 9,1 \\
\hline Durée du séjour \\
\hline Moyenne, jours & 8 & 3 & 0 & 5 \\
\hline Médiane & $3,0-14,0$ & $2,0-4,0$ & 0,0 & $3,0-12,0$ \\
\hline - EIQ 25-75 & $7(28,0)$ & $0(0,0)$ & $0(0,0)$ & $7(4,9)$ \\
\hline Soins intensifs & $0(0,0)$ & $0(0,0)$ & $0(0,0)$ & $0(0,0)$ \\
\hline Décès & $18(72,0)$ & $46(73,0)$ & $45(80,4)$ & $109(75,7)$ \\
\hline $\begin{array}{l}\text { Traitement aux } \\
\text { macrolides }\end{array}$ & & & & \\
\hline
\end{tabular}

Abréviations : EIQ, écart interquartile; n, nombre

a Tous les résultats sont donnés en fonction de $\mathrm{n}(\%)$, exception faite de la durée du séjour qui correspond à une moyenne (en jour) et à une médiane avec EIQ

\section{Discussion}

Cette étude dresse un bref portrait des manifestations et des issues cliniques des patients atteints de coqueluche qui se sont présentés dans un hôpital de soins pédiatriques tertiaires. Malgré l'adoption d'un programme universel d'immunisation contre la coqueluche au Québec, les bébés de moins de trois mois contractent encore la coqueluche. La plupart d'entre eux ont été atteints d'une forme grave de la maladie (84\%) et ont dû être hospitalisés (92\%), notamment à l'unité de soins intensifs (28\%), pour une durée médiane de huit jours, ce qui est comparable aux études antérieures $(10,18,21,25-31)$. La maladie était plus bénigne chez les enfants plus âgés, comme le démontrent les scores à l'ÉMP et les taux d'hospitalisation moins élevés. Seul un petit nombre de patients de 10 ans et plus avait contracté une coqueluche grave, aucun nécessitant une hospitalisation, ce qui donne à penser que les enfants plus âgés, tout comme les adultes, sont moins gravement touchés par cette infection $(25,32)$.

De façon générale, les symptômes évocateurs de la coqueluche, comme la toux paroxystique, le chant du coq et la toux émétisante, ont été observés chez une grande proportion d'enfants de tous âges, mais moins souvent que ce qui avait été déclaré précédemment $(25,29,31)$. Par exemple, lors d'études antérieures menées auprès d'enfants de neuf ans ou moins, la toux paroxystique, le chant du coq et la toux émétisante étaient signalés dans une proportion de 89 à $93 \%$ (toux), de 69 à $92 \%$ (chant du coq) et de 48 à $60 \%$ (toux émétisante) des enfants. En revanche, dans notre étude, ces symptômes étaient présents chez $76 \%$ (toux), $25 \%$ (chant du coq) et $48 \%$ (toux émétisante) des enfants du même âge. Ces variations pourraient être en partie attribuables à une atténuation de la maladie suivant I'immunisation, mais ils pourraient également être causés par une sensibilité plus élevée de la PCR par rapport à la culture, ce 
qui permet une détection des cas moins symptomatiques (33). II a précédemment été démontré que l'immunité et la protection que confère le vaccin acellulaire diminuent rapidement (9), ce qui pourrait expliquer la proportion élevée de résultats positifs chez les enfants de 10 à 15 ans (25-40\%).

Cette étude propose une description des cas de coqueluche chez les enfants au Québec. II y a plusieurs limites dont il faut tenir compte. Premièrement, cette étude se limitait à un seul centre; néanmoins, les 144 cas analysés dans l'étude représentaient environ $12,4 \%$ de tous les cas de coqueluche chez les moins de 18 ans diagnostiqués dans la province du Québec pendant la période étudiée (8). Deuxièmement, il existait un risque lié au biais d'information, notamment une mauvaise classification possible de l'état vaccinal, un risque intrinsèque associé à l'examen des dossiers. Nous avons essayé de minimiser le risque grâce à une concordance élevée entre les évaluateurs. Troisièmement, notre analyse a uniquement porté sur les patients qui ont consulté un médecin en milieu hospitalier, ce qui pourrait se traduire par une surestimation de la gravité de la maladie. Cependant, ce biais est probablement non significatif pour les nourrissons de moins de trois mois, puisque les cas de coqueluche déclarés dans ce groupe d'âge consultent habituellement les services d'urgence et sont hospitalisés (34). Pour terminer, la période étudiée a compris le sommet d'un cycle épidémique de quatre ans (2016), ce qui est susceptible d'occasionner une légère surestimation de l'incidence de la coqueluche.

Pour ce qui est des prochaines étapes et de la recherche à venir, cette étude pourrait constituer un point de référence pour une évaluation future des répercussions de la vaccination des femmes enceintes sur le fardeau de la coqueluche chez les jeunes enfants.

\section{Conclusion}

La coqueluche touche encore des enfants de tous âges au Québec. Chez les enfants plus âgés, la maladie est souvent plus bénigne. Chez les nourrissons qui ne bénéficient pas encore de la protection complète offerte par la vaccination, la maladie est souvent grave, en particulier chez ceux de moins de trois mois. Ces données probantes viennent appuyer davantage la mise en œuvre d'un programme de vaccination contre la coqueluche chez les femmes enceintes et fournissent une base de référence pour évaluer les effets de ce programme.

\section{Déclaration des auteurs}

M. D. ${ }^{\dagger}$ - conceptualisation, méthodologie, validation, enquête, organisation des données, rédaction de l'ébauche initiale, révision et édition de la version définitive D. I. ${ }^{\dagger}$ - conceptualisation, méthodologie, validation, analyse formelle, enquête, organisation des données, rédaction de l'ébauche initiale, révision et édition de la version définitive S. M. - méthodologie, révision et édition de la version définitive P. D. P. - analyse formelle, révision et édition de la version définitive

N. B. - méthodologie, révision et édition de la version définitive F. R. - enquête, ressources, révision et édition de la version définitive
C. Q. - conceptualisation, méthodologie, révision et édition de la version définitive, supervision, obtention des fonds

† M. D. et D. I. ont contribué en parts égales au travail.

\section{Conflit d'intérêts}

Aucun.

\section{Financement}

La Dre Quach a reçu un soutien salarial externe (FRQ-S merit, subvention no 252775). Ces travaux de recherche ont été rendus possibles par le Centre de recherche du CHU Sainte-Justine (fonds de démarrage de C. Quach).

\section{Références}

1. Bennett JE, Dolin R, Blaser MJ. Mandell, Douglas, and Bennett's Principles and Practice of Infectious Diseases. 8th Edition. Elsevier, 2014.

2. Gabutti G, Azzari C, Bonanni P, Prato R, Tozzi AE, Zanetti A, Zuccotti G. Pertussis. Hum Vaccin Immunother 2015;11(1):10817. https://doi.org/10.4161/hv.34364. PubMed (https://www. ncbi.nlm.nih.gov/pubmed/25483523?dopt=Abstract)

3. Health and Welfare Canada. National Advisory Committee on Immunization. A Guide to Immunization for Canadians. Dixon JMS, editor. Ottawa ON: Supply and Services Canada; 1980.

4. Smith T, Rotondo J, Desai S, Deehan H. Surveillance de la coqueluche au Canada : tendances jusqu'à 2012. Relevé des maladies transmissibles au Canada 2014 Fév;40(3):21-30. PubMed (https://www.ncbi.nlm.nih.gov/ pubmed/29769879?dopt=Abstract)

5. Douville-Fradet M, Amini R. Épidémiologie descriptive des maladies à déclaration obligatoire évitables par la vaccination au Québec, 2011-2013. Québec, Canada: Insitut National de Santé Publique; 2016.

6. Brousseau N, Judd L, Marcoux-Huard C, Landry M. Guide d'intervention - La coqueluche, mise à jour octobre 2016. Québec, Canada: MSSS; 2016. www.inspq.qc.ca/sites/default/ files/publications/2198_maladies_evitables_vaccination_ declaration_obligatoire.pdf

7. Kiely M, Boulianne N, Ouakki M, Audet D, Gariépy MC, Guay $M$, De Serres G, Dubé E. Enquête sur la couverture vaccinale des enfants de 1 an et 2 ans au Québec en 2016. Québec, Canada: INSPQ; 2017. www.inspq.qc.ca/sites/default/ files/publications/2341_enquete_couverture_vaccinale_ enfants_2016.pdf

8. Ministère de la santé et des services sociaux du Québec. Vigie et surveillance des maladies à déclaration obligatoire d'origine infectieuse Québec: MSSS; 2017. http://publications.msss. gouv.qc.ca/msss/fichiers/2017/17-268-05W.pdf

9. Schwartz KL, Kwong JC, Deeks SL, Campitelli MA, Jamieson FB, Marchand-Austin A, Stukel TA, Rosella L, Daneman N, Bolotin S, Drews SJ, Rilkoff H, Crowcroft NS. Effectiveness of pertussis vaccination and duration of immunity. CMAJ 2016 Nov;188(16):E399-406. https://doi.org/10.1503/cmaj.160193. PubMed (https://www.ncbi.nlm.nih.gov/pmc/articles/ PMC5088088/) 
10. Heininger $U$, Weibel D, Richard JL. Prospective nationwide surveillance of hospitalizations due to pertussis in children, 2006-2010. Pediatr Infect Dis J 2014 Feb;33(2):147-51. https://doi.org/10.1097/01. inf.0000435503.44620.74. PubMed (https://www.ncbi.nlm.nih. gov/pubmed/24413406?dopt=Abstract)

11. Hviid A, Stellfeld M, Andersen PH, Wohlfahrt J, Melbye M. Impact of routine vaccination with a pertussis toxoid vaccine in Denmark. Vaccine 2004 Sep;22(27-28):3530-4. https://doi. org/10.1016/j.vaccine.2004.03.046. PubMed (https://www. ncbi.nlm.nih.gov/pubmed/15315832?dopt=Abstract)

12. Gall SA, Myers J, Pichichero M. Maternal immunization with tetanus-diphtheria-pertussis vaccine: effect on maternal and neonatal serum antibody levels. Am J Obstet Gynecol 2011 Apr;204(4):334.e1-5. https://doi.org/10.1016/j. ajog.2010.11.024. PubMed (https://www.ncbi.nlm.nih.gov/ pubmed/21272845?dopt=Abstract)

13. Abu Raya B, Srugo I, Kessel A, Peterman M, Bader D, Gonen $\mathrm{R}$, Bamberger $\mathrm{E}$. The effect of timing of maternal tetanus, diphtheria, and acellular pertussis (Tdap) immunization during pregnancy on newborn pertussis antibody levels - a prospective study. Vaccine 2014 Oct;32(44):5787-93. https:// doi.org/10.1016/j.vaccine.2014.08.038. PubMed (https://www. ncbi.nlm.nih.gov/pubmed/25173476?dopt=Abstract)

14. Baxter R, Bartlett J, Fireman B, Lewis E, Klein NP. Effectiveness of Vaccination During Pregnancy to Prevent Infant Pertussis. Pediatrics 2017 May;139(5):e20164091. https://doi. org/10.1542/peds.2016-4091. PubMed (https://www.ncbi.nlm. nih.gov/pubmed/28557752?dopt=Abstract)

15. Comité consultatif national de l'immunisation. Mise à jour sur l'immunisation durant la grossesse avec le vaccin combiné anti-Tétanos, et à dose réduite contre la diphtérie et la coqueluche acellulaire (dcaT). Canada: Agence de la santé publique du Canada; 2018. www.canada.ca/en/public-health/ services/publications/healthy-living/update-immunizationpregnancy-tdap-vaccine.html

16. Comité sur l'immunisation du Québec $(\mathrm{ClO})$. Avis sur la pertinence de mettre en place la vaccination contre la coqueluche chez toutes les femmes enceintes au Québec. Québec: Institut national de santé publique du Québec; 2016. www.inspq.qc.ca/publications/2089

17. Halperin SA, Wang EE, Law B, Mills E, Morris R, Déry P, Lebel M, MacDonald N, Jadavji T, Vaudry W, Scheifele D, Delage G, Duclos P. Epidemiological features of pertussis in hospitalized patients in Canada, 1991-1997: report of the Immunization Monitoring Program--Active (IMPACT). Clin Infect Dis 1999 Jun;28(6):1238-43. https://doi. org/10.1086/514792. PubMed (https://www.ncbi.nlm.nih.gov/ pubmed/10451159?dopt=Abstract)

18. Bettinger JA, Halperin SA, De Serres G, Scheifele DW, Tam T. The effect of changing from whole-cell to acellular pertussis vaccine on the epidemiology of hospitalized children with pertussis in Canada. Pediatr Infect Dis J 2007 Jan;26(1):31-5. https://doi.org/10.1097/01. inf.0000247055.81541.04. PubMed (https://www.ncbi.nlm.nih. gov/pubmed/17195702?dopt=Abstract)

19. Direction des communications du CHU Sainte-Justine. Rapport annuel CHU Sainte-Justine 2016-2017. Montréal: CHU Sainte-Justine; 2017. www.chusj.org/CORPO/ files/3c/3cb7bfb5-02e6-4629-866e-5f9f3a815566.pdf

20. Liko J, Robison SG, Cieslak PR. Do Pertussis Vaccines Protect Against Bordetella parapertussis? Clin Infect
Dis 2017 Jun;64(12):1795-7. https://doi.org/10.1093/ cid/cix221 PubMed (https://www.ncbi.nlm.nih.gov/ pubmed/28369240?dopt=Abstract)

21. Cherry JD, Tan T, Wirsing von König CH, Forsyth KD, Thisyakorn U, Greenberg D, Johnson D, Marchant C, Plotkin S. Clinical definitions of pertussis: Summary of a Global Pertussis Initiative roundtable meeting, February 2011. Clin Infect Dis 2012 Jun;54(12):1756-64. https://doi.org/10.1093/ cid/cis302. PubMed (https://www.ncbi.nlm.nih.gov/ pubmed/22431797?dopt=Abstract)

22. Gill CJ, Mwananyanda L, MacLeod W, Kwenda G, Mwale M, Williams AL, Siazeele K, Yang Z, Mwansa J, Thea DM. Incidence of Severe and Nonsevere Pertussis Among HIV-Exposed and -Unexposed Zambian Infants Through 14 Weeks of Age: Results From the Southern Africa Mother Infant Pertussis Study (SAMIPS), a Longitudinal Birth Cohort Study. Clin Infect Dis 2016 Dec;63(suppl 4):S154-64. https://doi. org/10.1093/cid/ciw526. PubMed (https://www.ncbi.nlm.nih. gov/pubmed/27838668?dopt=Abstract)

23. Omer SB, Kazi AM, Bednarczyk RA, Allen KE, Quinn CP, Aziz F, Sial K, Phadke VK, Tondella ML, Williams MM, Orenstein WA, Ali SA. Epidemiology of Pertussis Among Young Pakistani Infants: A Community-Based Prospective Surveillance Study. Clin Infect Dis 2016 Dec;63 suppl 4:S148-53. https://doi. org/10.1093/cid/ciw561. PubMed (https://www.ncbi.nlm.nih. gov/pubmed/27838667?dopt=Abstract)

24. Soofie N, Nunes MC, Kgagudi P, van Niekerk N, Makgobo T, Agosti Y, Hwinya C, Pathirana J, Madhi SA. The Burden of Pertussis Hospitalization in HIV-Exposed and HIV-Unexposed South African Infants. Clin Infect Dis 2016 Dec;63 suppl 4:S165-73. https://doi.org/10.1093/ cid/ciw545. PubMed (https://www.ncbi.nlm.nih.gov/ pubmed/27838669?dopt=Abstract)

25. Kilgore PE, Salim AM, Zervos MJ, Schmitt HJ. Pertussis: Microbiology, Disease, Treatment, and Prevention. Clin Microbiol Rev 2016 Jul;29(3):449-86. https://doi.org/10.1128/ CMR.00083-15. PubMed (https://www.ncbi.nlm.nih.gov/ pubmed/27029594?dopt=Abstract)

26. Del Valle-Mendoza J, Casabona-Oré V, Petrozzi-Helasvuo V, Cornejo-Tapia A, Weilg P, Pons MJ, Cieza-Mora E, Bazán-Mayra J, Cornejo-Pacherres H, Ruiz J. Bordetella pertussis diagnosis in children under five years of age in the Regional Hospital of Cajamarca, Northern Peru. J Infect Dev Ctries 2015 Nov;9(11):1180-5. https://doi.org/10.3855/ jidc.6803. PubMed (https://www.ncbi.nlm.nih.gov/ pubmed/26623626?dopt=Abstract)

27. Masseria C, Martin CK, Krishnarajah G, Becker LK, Buikema A, Tan TQ. Incidence and Burden of Pertussis Among Infants Less Than 1 Year of Age. Pediatr Infect Dis J 2017 Mar;36(3):e54-61. https://doi.org/10.1097/ INF.0000000000001440. PubMed (https://www.ncbi.nlm.nih. gov/pubmed/27902648?dopt=Abstract)

28. Kaczmarek MC, Ware RS, McEniery JA, Coulthard MG, Lambert SB. Epidemiology of pertussis-related paediatric intensive care unit (ICU) admissions in Australia, 1997-2013: an observational study. BMJ Open 2016 Apr;6(4):e010386. https://doi.org/10.1136/ bmjopen-2015-010386. PubMed (https://www.ncbi.nlm.nih. gov/pubmed/27053270?dopt=Abstract)

29. Carlsson RM, von Segebaden K, Bergstrom J, Kling AM, Nilsson L. Surveillance of infant pertussis in Sweden 19982012; severity of disease in relation to the national vaccination 
programme. Euro Surveill 2015 Feb;20(6):21032. https://doi. org/10.2807/1560-7917.ES2015.20.6.21032. PubMed (https:// www.ncbi.nlm.nih.gov/pubmed/25695476?dopt=Abstract)

30. Cortese MM, Baughman AL, Zhang R, Srivastava PU, Wallace GS. Pertussis hospitalizations among infants in the United States, 1993 to 2004. Pediatrics 2008 Mar;121(3):484-92. https://doi.org/10.1542/peds.2007-1393. PubMed (https:// www.ncbi.nlm.nih.gov/pubmed/18310196?dopt=Abstract)

31. Gentile A, Romanin VS, Juárez MV, Lución MF, Marques $\mathrm{ML}$, Mistchenko AS. Epidemiology of Bordetella pertussis in a children's hospital. Arch Argent Pediatr 2014 Feb;112(1):26-32. https://doi.org/10.1590/S032500752014000100006. PubMed (https://www.ncbi.nlm.nih.gov/ pubmed/24566778?dopt=Abstract)
32. Kline JM, Lewis WD, Smith EA, Tracy LR, Moerschel SK. Pertussis: a reemerging infection. Am Fam Physician 2013 Oct;88(8):507-14. PubMed (https://www.ncbi.nlm.nih.gov/ pubmed/24364571?dopt=Abstract)

33. Kaczmarek MC, Ware RS, Lambert SB. The contribution of PCR testing to influenza and pertussis notifications in Australia. Epidemiol Infect 2016 Jan;144(2):306-14. https://doi. org/10.1017/S0950268815001004. PubMed (https://www.ncbi. nlm.nih.gov/pubmed/26112983?dopt=Abstract)

34. Winter K, Cherry JD, Harriman K. Effectiveness of Prenatal Tetanus, Diphtheria, and Acellular Pertussis Vaccination on Pertussis Severity in Infants. Clin Infect Dis 2017 Jan;64(1):914. https://doi.org/10.1093/cid/ciw633. PubMed (https://www. ncbi.nlm.nih.gov/pubmed/27624956?dopt=Abstract)

\section{RMTC INFOGRAPHIE}

L'immunité conférée par le vaccin contre la coqueluche diminue avec le temps

La coqueluche est une infection des voies respiratoires qu'on peut prévenir par la vaccination.

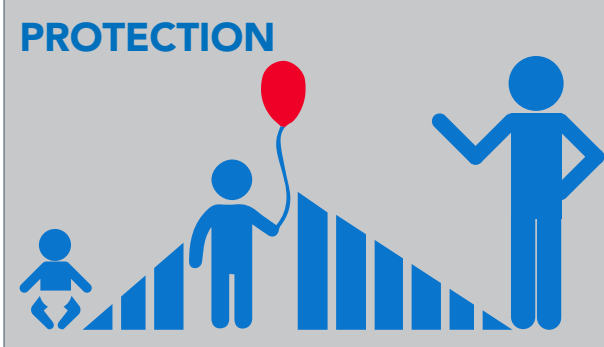

Toutefois, il faut plusieurs injections pour qu'une protection complète soit conférée. Cette protection s'atténue avec le temps.

\section{LA COQUELUCHE DEMEURE DANGEREUSE POUR LES NOURRISSONS}

\section{Les nourrissons sont les plus à risque}

Les nourrissons de moins de trois mois n'ont reçu qu'une seule dose du vaccin.

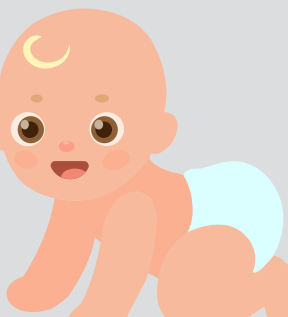

Les nourrissons sont plus

\section{sévèrement atteints}

Une étude récente suggère que les nourrissons de moins de trois mois qui sont infectés par la coqueluche développent une maladie sévère. Parmi ceux-ci1:

- $92 \%$ ont dû être hospitalisés

- $28 \%$ ont été admis aux soins intensifs

\section{Pour prévenir l'infection}

Assurez-vous de vous tenir votre vaccination et celle de famille à jour²:

1. CALENDRIER DE VACCINATION SYSTÉMATIQUE POUR LES ENFANTS ET LES NOURRISSONS

- A 2, 4, 6, 12-23 mois et 4-6 ans

2. DOSE DE RAPPEL À L'ADOLESCENCE À $14-16$ ans

\section{DOSE DE RAPPEL À L'ÂGE ADULTE}

\section{4. À CHAQUE GROSSESSE}

- Des anticorps protecteurs sont transférés au foetus - Optimalement entre 27-32 semaines

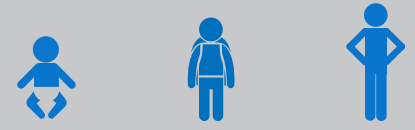

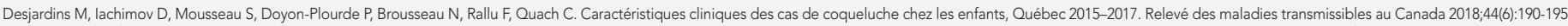
${ }^{2}$ Comité consultatif national de l'immunisation (CCNI). Vaccin contre la coqueluche Partie 4 - Agents d'immunisation active. Mars 2018. Ottawa : Agence de la santé publique du Canada. https://www.canada.ca/fr/sante-publique/services/ publications/vie-saine/guide-canadien-immunisation-partie-4-agents-immunisation-active/page-15-vaccin-contre-coqueluche.html

Citation proposée : La coqueluche demeure dangereuse pour les nourrissons. Relevé des maladies transmissibles au Canada 2018 44(9):220. 